International Journal of Information Acquisition

Vol. 5, No. 4 (2008) 369-370

(c) World Scientific Publishing Company

\title{
AUTHOR INDEX Volume 5 (2008)
}

Aizawa, N., see Kubota, N.

Bellotto, N. \& Hu, H., Multimodal

People Tracking and Identification

for Service Robots

Bistry, H., see Maeder, A.

Brunete, A., Torres, J. E., Hernando, M. \& Gambao, E., A Proposal for a Multi-Drive Heterogeneous Modular Pipe-Inspection

Micro-Robot

Buss, M., see $\mathrm{Xu}, \mathrm{T}$.

Chaen, T., see Yu, Y.

Chen, Q., see Zhang, H.

Chou, T.-L., Fujii, N., Ota, J., Tanaka, M. \& Shinozaki, A., Low-Cost and Robust Positioning System for Indoor Group of Forklifts Utilizing Probability Estimation and Sensor Network de Silva, C. W., see Lang, H.

Ding, H., see Li, Y.

Elfadil, N., Knowledge Extraction from Rise-Time Auto-Correlated Patterns

Fujii, N., see Chou, T.-L.

Gambao, E., see Brunete, A.

$\mathrm{Gu}$, D., see Samperio, R.

Guan, Y.-P., Automatic Extraction of Lip Based on Lip Map

Guo, J., see Wang, J.

Guo, S., see Wang, J.

Han, L., see Zheng, Z.

Hernando, M., see Brunete, A.

Hong, R., see Li, S.

$\mathrm{Hu}, \mathrm{H}$., see Bellotto, N.

$\mathrm{Hu}, \mathrm{H}$., see Samperio, R.

Ioffe, A., see Ma, G.

Iwasa, K., see Narita, M.

Kamo, Y., see $\mathrm{Yu}, \mathrm{Y}$.

Kawakoe, H., see Yu, Y. $\begin{array}{lll}\mathbf{3} & (2008) & 247 \\ & & \\ \mathbf{3} & (2008) & 209 \\ \mathbf{3} & (2008) & 259 \\ & & \\ & \\ \mathbf{2} & (2008) & 111 \\ \mathbf{4} & (2008) & 309 \\ \mathbf{3} & (2008) & 197 \\ \mathbf{3} & (2008) & 283\end{array}$

2 (2008) 163

2 (2008) 93

4 (2008) 349

2 (2008) 181

2 (2008) 163

2 (2008) 111

4 (2008) 331

1 (2008) 31

1 (2008) 83

1 (2008) 83

1 (2008) 41

2 (2008) 111

3 (2008) 235

3 (2008) 209

4 (2008) 331

1 (2008) 11

2 (2008) 127

4 (2008) 359

4 (2008) 359
Kim, S. \& Lee, S., Robust Mobile Robot Velocity Estimation Using a Polygonal Array of Optical Mice

Kim, Y. K., see Yang, X.

Kim, Y. K., see Yang, X.

Kojima, H., see Kubota, N.

Kondo, H., see Wang, J.

Kubota, N., Kojima, H., Aizawa, N. \& Tang, D., Dynamic Topological Visualization of Change in Perceptual Information of Partner Robots

Kühnlenz, K., see Xu, T.

Kummert, A., see Ma, G.

Lang, H. \& de Silva, C. W., Fault Diagnosis of an Industrial Machine Through Sensor Fusion

Lawson, R. P. W., see Ma, Y.

Lee, S., see Kim, S.

Li, S., Hong, R., Liu, B. \& Wu, X., A Quality Metric Based on Color Similarity for Image Fusion

Li, Y., Tang, Z., Ding, H. \& Zhang, Y., Discriminative Feature Extraction Based on Self-Adaptive Frequency Warping for Robust Speaker Identification

Liu, B., see Li, S.

Liu, R. \& Meng, M. Q. H., Time-to-Contact Information Estimation for Monocular Mobile Robots

4 (2008) 321

2 (2008) 149

3 (2008) 269

3 (2008) 247

1 (2008) 83

3 (2008) 247

4 (2008) 309

1 (2008) 11

2 (2008) 93

3 (2008) 189

4 (2008) 321

3 (2008) 235

4 (2008) 349

3 (2008) 235

3 (2008) 223

Low, K. H., Zhou, C., Seet, G. G. L. \& Yu, J., Learning from

Gymnotiform Swimmers - Design and Implementation of Robotic

Knifefish NKF-II

2 (2008) 137 Müller-Schneiders, S. \& Kummert, A., A Real-Time Detection 
Algorithm for Vision-Based Pedestrian Protection

Ma, Y., Lawson, R. P. W. \&

Robinson, A. M., High Sensitivity and High Dynamic Range Optical Micro-Radiator Vacuum Sensor

Fabricted with CMOS Technology

Maeder, A., Bistry, H. \& Zhang, J., Intelligent Vision Systems for

Robotic Applications

Meng, M. Q. H., see Liu, R.

Meng, M. Q.-H., see Xu, L.

Mohammad, Y. \& Nishida, T., Interaction Between Untrained Users and a Miniature Robot in a Collaborative Navigation

Controlled Experiment

Müller-Schneiders, S., see Ma, G.

Narita, M., Shimamura, M., Iwasa, K. \& Yamaguchi, T., Interoperability Verification for Web Service Based Robot Communication Platforms and Contribution to the International Standards

Nishida, T., see Mohammad, Y.

Ota, J., see Chou, T.-L.

$\mathrm{Ou}, \mathrm{Y}$., see $\mathrm{Wu}, \mathrm{X}$.

Park, S.-B., see Ma, G.

Qian, H., see Wu, X.

Ren, H., see Xu, L.

Robinson, A. M., see Ma, Y.

Samperio, R., Hu, H. \& Gu, D., Implementation of a Localization-Oriented HRI for Walking Robots in the RoboCup Environment

4 (2008) 331

2 (2008) 137

2 (2008) 127

2 (2008) 163

1 (2008) 51

1 (2008) 83

2 (2008) 163

3 (2008) 247

4 (2008) 349

2 (2008) 111

3 (2008) 197

4 (2008) 359
Operating System with Force

Feedback for Medical Applications 1 (2008) 83

Wang, Z., see Zheng, Z.

Winzer, P., see Sitte, J.

Wu, X., Ou, Y., Qian, H. \& Xu, Y., A Real Time Face Classification and Counting System

$\mathrm{Wu}, \mathrm{X}$., see Li, S.

Xu, L., Meng, M. Q.-H. \& Ren, H., Applicability of Homogeneous Human Trunk Phantom in Estimating the Radiation Characteristics of Body-Worn Devices

1 (2008) 41

1 (2008) 51

1 (2008) 1

3 (2008) 235

1 (2008) 65

Xu, T., Kühnlenz, K. \& Buss, M., A Multi-Camera View Direction Planning Strategy for Mobile Robots

$\mathrm{Xu}, \mathrm{Y}$., see Wu, X.

Yamaguchi, T., see Narita, M.

Yan, H., see Zhang, H.

Yang, X. \& Kim, Y. K., Hand Manipulation Training in Haptic Virtual Environments

Yang, X., Zhao, Z. \& Kim, Y. K., A Real Time Traffic Light Recognition System

$\mathrm{Yu}$, J., see Low, K. H.

Yu, Y., Chaen, T. \& Tsujio, S., High-Stiffness and High-Sensitivity 3-Axis Force Sensor Using Strain-Deformation Expansion Mechanism

Yu, Y., Kamo, Y., Kawakoe, H. \& Tsujio, S., Estimation of Contact Shape of Quadric Environment with Object Probing Operation

Zha, Z., see Zheng, Z.

Zhang, H., Chen, Q. \& Yan, H., Decentralized Robust Control with Guaranteed Cost for Hybrid Complex Networks

Zhang, J., see Maeder, A.

Zhang, Y., see Li, Y.

Zhao, Z., see Yang, X.

Zheng, Z., Zha, Z., Han, L. \& Wang, Z., Feature Detection and Correspondence for Camera Calibration

Zhou, C., see Low, K. H.
3 (2008) 197

1 (2008) 41

4 (2008) 309

1 (2008) 1

2 (2008) 127

3 (2008) 283

3 (2008) 269

2 (2008) 149

2 (2008) 137

4 (2008) 359

1 (2008) 41

3 (2008) 283

3 (2008) 259

4 (2008) 349

2 (2008) 149

2 (2008) 137

Tsujio, S., see Yu, Y.
Wang, J., Guo, S., Kondo, H., Guo, J. \& Tamiya, T., A Novel Catheter 